\title{
Quick check for tumour infiltration
}

Tumour infiltration into normal brain tissue in humans can be reliably detected with stimulated Raman scattering (SRS) microscopy, according to a new study. The technique could improve surgical accuracy and treatment outcomes.

Incomplete resection of tumourinfiltrated brain tissue commonly leads to tumour regrowth. "However, no modality can track such infiltration rapidly in unprocessed tissues," says Daniel Orringer, a senior author on the new study.

A team led by Sunney Xie, who first developed the technique, previously showed that SRS microscopy could delineate tumours from healthy tissue in animals. The imaging requires no tissue staining, but exploits the properties of lipids and proteins to distinguish between cell bodies and axons: tumours exhibit hypercellularity and low axonal density. Orringer, Xie and colleagues have now used SRS microscopy to image healthy tissue and tumours from 22 humans.
The SRS microscopy images enabled pathologists to identify normal tissue, infiltrating glioma and dense glioma with the same reliability as they could with haematoxylin and eosin staining. The team also developed a system to automatically quantify the probability of tumour infiltration in a specific area on the basis of the proteinlipid ratio, cellularity and axonal density in that area. The system identified infiltrated tissue with a sensitivity of $97.5 \%$ and a specificity of $98.5 \%$.

"This technology could take some of the guesswork out of brain tumour surgery," says Orringer. "I think we'll be relying on an FDA-approved SRS microscope for intraoperative resection margin control within 18-24 months."

Ian Fyfe

Original article Ji, M. et al. Detection of human brain tumor infiltration with quantitative stimulated Raman scattering microscopy. Sci. Transl. Med. doi:10.1126/ scitranslmed.aab0195 